\title{
WEAK SOLUTIONS OF HYPERBOLIC-PARABOLIC VOLTERRA EQUATIONS
}

\author{
GUSTAF GRIPENBERG
}

\begin{abstract}
The existence of a global weak solution, satisfying certain a priori $L^{\infty}$-bounds, of the equation $u_{t}(t, x)=\int_{0}^{t} k(t-s)\left(\sigma\left(u_{x}\right)\right)_{x}(s, x) d s+f(t, x)$ is established. The kernel $k$ is locally integrable and log-convex, and $\sigma^{\prime}$ has only one local minimum which is positive.
\end{abstract}

\section{INTRODUCTION}

The purpose of this paper is to establish the existence of a global weak solution of the equation

(1)

$$
\begin{aligned}
u_{t}(t, x) & =\int_{0}^{t} k(t-s)\left(\sigma\left(u_{x}\right)\right)_{x}(s, x) d s+f(t, x), \quad t \geq 0, x \in(0,1), \\
u(0, x) & =u_{0}(x), \quad x \in[0,1] \\
u(t, 0) & =u(t, 1)=0, \quad t>0
\end{aligned}
$$

(where the subscripts denote differentiation with respect to the corresponding variables), and to show that this weak solution satisfies certain a priori $L^{\infty}$. bounds.

Equation (1) is of interest because it appears in mathematical models of viscoelasticity (see [18 and 31]), but also because it is of a form somewhere between a parabolic and a hyperbolic equation. This is due to the fact that when $k \equiv 1$ we get the nonlinear wave equation

$$
u_{t t}(t, x)=\left(\sigma\left(u_{x}\right)\right)_{x}(t, x)+f_{t}(t, x),
$$

and if, on the other hand, $k$ is replaced by the delta functional, then we get the nonlinear diffusion equation

$$
u_{t}(t, x)=\left(\sigma\left(u_{x}\right)\right)_{x}(t, x)+f(t, x) .
$$

The nonlinear wave equation (that corresponds to the completely elastic case in mechanics) has a weak solution with both $u_{x}$ and $u_{t}$ bounded and initially smooth solutions develop singularities (in the derivatives) in finite time. The

Received by the editors September 22, 1992.

1991 Mathematics Subject Classification. Primary 45K05; Secondary 45D05, 45N05, 35K99, 35L99, 73 F99.

Key words and phrases. Volterra equation, weak solution, $L^{\infty}$-bound, viscoelasticity, parabolic, hyperbolic. 
same results hold for (1) at least in the case where $k^{\prime}$ is locally of bounded variation on $\mathbb{R}^{+}$, see [27] for the global existence of a weak solution and see $[2,9,15,26]$ for the breakdown of smooth solutions.

The diffusion equation (that corresponds to the completely viscous case) has always (under reasonable assumptions on $\sigma$ ) a strong solution and using the maximum principle it is easy to show that $u_{x}$ remains bounded. But in contrast to the wave equation, the variables $t$ and $x$ are no longer symmetrical and boundedness of $u_{t}$ would for the diffusion equation imply that $u_{x x}$ is bounded as well. It may therefore be interesting to see in greater detail how the symmetry between the variables breaks down when one goes from the wave equation to the diffusion equation.

In [11] the existence of a strong solution of (1) is established for kernels $k$ that satisfy

$$
\Re \hat{k}(z) \geq \kappa|\Im \hat{k}(z)|, \quad \Re z>0,
$$

(a typical example is $k(t)=t^{-\alpha}$ in which case $\kappa=\tan (\alpha \pi / 2)$ ) but then the nonlinearity is quantitatively restricted by the positive constant $\kappa$. Condition (2) is also used in [29 and 30], and it seems to be a very natural assumption. In [12] strong solutions are furthermore shown to exist under the assumption that that $k$ is nonincreasing and convex, and $\liminf _{t \downarrow 0} \sqrt{t} k(t)>0$, i.e., $k$ is more singular at 0 than $t^{-1 / 2}$.

In [5 and 22] weak solutions of equations that contain (1) as a special case are studied. For equation (1) it is required that $k(0)=1$ and $k^{\prime}(0+)=-\infty$ and less is assumed of the nonlinearity $\sigma$ than in this paper. In [23] a weak solution is found when $k$ satisfies a (rather complicated but not very restrictive) frequency domain condition and there is no requirement that $k(0)=1$.

The purpose of this paper is to show how one can find weak solutions of all equations between the wave and diffusion equations such that $u_{x}$ remains bounded but the boundedness of $u_{t}$ in the wave equation turns into the boundedness of $\frac{d}{d t} \int_{0}^{t}(t-s)^{-1 / 2}\left(u(s, x)-u_{0}(x)\right) d s$ for the diffusion equation (that is not formally included in (1) although it would be easy to add a term $\sigma\left(u_{x}\right)_{x}$ outside the integral). But note that at the moment a major weakness in the theory is the lack of reasonable uniqueness results.

The argument used here is not able to capture the fact that weak solutions at some point turn into strong ones and therefore one of the main points of this paper is that we get a priori $L^{\infty}$-bounds on the weak solution, something that is not achieved in, e.g., [5, 22], or [23], nor for the strong solutions found in [11 and 12]. In the case where $\left|k^{\prime}(0+)\right|<\infty$ this paper does not offer any significantly better results than those that can to be found in [27].

Another approach to studying (1) and related equations is to try to find smooth solutions for sufficiently small initial data (or for a finite time only). There is a wealth of results in this direction, see, e.g., $[1,3,17,20,21,24,25$, $29,30,32]$. In many of these papers the equation studied is

$$
u_{t t}(t, x)=\phi\left(u_{x}(t, x)\right)_{x}+\int_{0}^{t} a(t-s) \psi\left(u_{x}(s, x)\right)_{x} d s+g(t, x),
$$

which is the same as (1) if $\phi=\psi=\sigma, k(0)=1$, and $a=k^{\prime}$. In the case where $\phi$ is a linear function, the existence of global solutions for arbitrary data is established in [16]. 
For linear equations, one difference between the hyperbolic wave equation and the parabolic diffusion equation is that in the hyperbolic case the solutions have a finite speed of propagation and discontinuities are not smoothed out. For Volterra equations one can prove (see $[4,19,28]$ ) that the finite speed of propagation is related to the assumption that $k(0+)<\infty$ and that discontinuities are smoothed out if $k^{\prime}(0+)=-\infty$. In the special case $k(t)=t^{-\alpha}$, $\alpha \in(0,1)$, one gets a more detailed interpolation result, see [6 and 7].

\section{STATEMENT OF RESUlTS}

In order to be able to formulate our result, we first have to describe how to rewrite (1) in a form better suited for the analysis. This reformulation of the equation is, of course, the crucial idea in this paper.

We replace the boundary conditions $u(t, 0)=u(t, 1)=0$ by the requirement that $u(t, \bullet)$ should be an odd periodic function with period 2 and therefore we have to extend $u_{0}$, and $f(t, \bullet)$ to be of this type. Let us proceed quite formally, without worrying about differentiability, etc., and take $y=u_{t}$ and $w=u_{x}$. Then (1) becomes

$$
\begin{aligned}
w_{t}(t, x) & =y_{x}(t, x), \\
y(t, x) & =\int_{0}^{t} k(t-s)(\sigma(w))_{x}(s, x) d s+f(t, x) .
\end{aligned}
$$

Let $a$ be the solution of the equation

$$
\frac{1}{k(0+)} k(t)+\int_{0}^{t} a(t-s) k(s) d s=1, \quad t>0 .
$$

It follows that the system of equations can be rewritten as

$$
\begin{aligned}
& w_{t}(t, x)=y_{x}(t, x), \\
& \frac{\partial}{\partial t}\left(\frac{1}{k(0)}(y(t, x)-f(t, x))+\int_{0}^{t} a(t-s)(y(s, x)-f(s, x)) d s\right) \\
& =(\sigma(w))_{x}(t, x) .
\end{aligned}
$$

Take $\gamma=1 / \sqrt{k(0)}$ and let $b$ be the solution of the equation

$$
2 \gamma b(t)+\int_{0}^{t} b(t-s) b(s) d s=a(t), \quad t>0,
$$

in other words, $\gamma \delta+b$ is the convolution square root of $\gamma^{2} \delta+a$ (where $\delta$ denotes the unit point mass a zero). Moreover, we define the function $v$ to be

$$
v(t, x)=\gamma y(t, x)+\int_{0}^{t} b(t-s) y(s, x) d s .
$$

When we take the convolution of both sides of (4) with $\gamma \delta+b$, the system of equations thus becomes

(6)

$$
\begin{aligned}
& \frac{\partial}{\partial t}\left(\gamma(w(t, x)-w(0, x))+\int_{0}^{t} b(t-s)(w(s, x)-w(0, x)) d s\right)=v_{x}(t, x), \\
& \frac{\partial}{\partial t}\left(\gamma(v(t, x)-g(t, x))+\int_{0}^{t} b(t-s)(v(s, x)-g(s, x)) d s\right)=(\sigma(w))_{x}(t, x)
\end{aligned}
$$


where

$$
g(t, x)=\gamma f(t, x)+\int_{0}^{t} b(t-s) f(s, x) d s .
$$

This is the system we shall primarily solve, or more precisely, we will replace this system of equations with the following reguiarized version involving a parameter $\epsilon$ and then we let $\epsilon \downarrow 0$ :

$$
\begin{aligned}
& \frac{\partial}{\partial t}\left(\gamma\left(w^{\epsilon}(t, x)-w_{0}^{\epsilon}(x)\right)+\int_{0}^{t} b(t-s)\left(w^{\epsilon}(s, x)-w_{0}^{\epsilon}(x)\right) d s\right) \\
& =v_{x}^{\epsilon}(t, x)+\epsilon w_{x x}^{\epsilon}(t, x), \quad w^{\epsilon}(0, x)=w_{0}^{\epsilon}(x), \\
& \frac{\partial}{\partial t}\left(\gamma\left(v^{\epsilon}(t, x)-g^{\epsilon}(t, x)\right)+\int_{0}^{t} b(t-s)\left(v^{\epsilon}(s, x)-g^{\epsilon}(s, x)\right) d s\right) \\
& =\left(\sigma\left(w^{\epsilon}\right)\right)_{x}(t, x)+\epsilon v_{x x}^{\epsilon}(t, x), \quad v^{\epsilon}(0, x)=g^{\epsilon}(0, x),
\end{aligned}
$$

where $t \geq 0, x \in \mathbb{R}, w^{\epsilon}$ is an even and $v^{\epsilon}$ an odd function in the variable $x$, both periodic with period 2 . Here we have replaced $w(0, \bullet)=u_{0 x}$ and $g$ by a more regular functions $w_{0}^{\epsilon}$ and $g^{\epsilon}$, respectively, in order to avoid unnecessary technical difficulties in establishing the existence of a sufficiently smooth solution of (8).

We need the following results about the kernels $a, b$, and the solution $c$ of the equation

$$
\gamma c(t)+\int_{0}^{t} b(t-s) c(s) d s=1, \quad t>0 .
$$

Lemma 1. Assume that $k \in L_{\mathrm{loc}}^{1}\left(\mathbb{R}^{+} ; \mathbb{R}\right)$ is positive, nonincreasing, and logconvex on $(0, \infty)$. Then there are solutions $a, b$, and $c \in L_{\text {loc }}^{1}\left(\mathbb{R}^{+} ; \mathbb{R}\right)$ of (3), (5), and (9), respectively, and they are nonnegative and nonincreasing on $(0, \infty)$. Moreover, $b(0+)=\infty$ if and only if $\left|k^{\prime}(0+)\right|=\infty, c(0+)=\sqrt{k(0+)}$ and

$$
\int_{0}^{t} c(t-s) c(s) d s=\int_{0}^{t} k(s) d s, \quad t \geq 0 .
$$

We may, without loss of generality, assume that $b$ is left-continuous on $(0, \infty)$ so that we have $b(t)=b(\infty)+\beta([t, \infty))$, where $\beta$ is a nonnegative measure.

By $W^{m, p}(I ; X)$ we denote the space of functions: $I \rightarrow X$ that are $m$ times differentiable (i.e., the function is the integral of its derivative) and are such that the function and the $m$ first derivatives belong to $L^{p}(I ; X)$. These spaces are used for notational convenience and no results from the more advanced theory of Sobolev spaces are used. Furthermore, we use the convention that a subindex $e$ indicates that the function is even and the subindex $o$ that it is odd. Furthermore, $\mathbb{T}$ denotes the real axis with the points $x$ and $x+2$ identified. Thus a function $\mathbb{T} \rightarrow \mathbb{R}$ can be identified with a function $\mathbb{R} \rightarrow \mathbb{R}$ which is periodic with period 2 .

First we state a technical result that says that there is a sufficiently nice solution of (8). 
Lemma 2. Let $\epsilon>0$ and assume that

(i) $k \in L_{\text {loc }}^{1}\left(\mathbb{R}^{+} ; \mathbb{R}\right)$ is positive and nonincreasing on $(0, \infty)$ and $\log (k)$ is convex on this interval;

(ii) $\sigma \in C^{1}(\mathbb{R} ; \mathbb{R})$ and $0<\inf _{s \in \mathbb{R}} \sigma^{\prime}(s) \leq \sup _{s \in \mathbb{R}} \sigma^{\prime}(s)<\infty$;

(iii) $g^{\epsilon} \in W_{\text {loc }}^{1,1}\left(\mathbb{R}^{+} ; L_{o}^{\infty}(\mathbb{T} ; \mathbb{R})\right) \cap W_{\text {loc }}^{1,2}\left(\mathbb{R}^{+} ; L_{o}^{2}(\mathbb{T} ; \mathbb{R})\right)$ and $g^{\epsilon}(0, \bullet) \in$ $W_{o}^{2,2}(\mathbb{T} ; \mathbb{R}) ;$

(iv) $w_{0}^{\epsilon} \in W_{e}^{2,2}(\mathbb{T} ; \mathbb{R})$.

Then there exists a solution $\left(w^{\epsilon}, v^{\epsilon}\right)$ of $(8)$ such that

$$
w^{\epsilon} \in L_{\mathrm{loc}}^{1}\left(\mathbb{R}^{+} ; W_{e}^{2,2}(\mathbb{T} ; \mathbb{R})\right) \cap B V_{\mathrm{loc}}\left(\mathbb{R}^{+} ; L_{e}^{2}(\mathbb{T} ; \mathbb{R})\right) \cap L_{\mathrm{loc}}^{\infty}\left(\mathbb{R}^{+} ; W_{e}^{1,2}(\mathbb{T} ; \mathbb{R})\right),
$$

and

$$
v^{\epsilon} \in L_{\mathrm{loc}}^{1}\left(\mathbb{R}^{+} ; W_{o}^{2,2}(\mathbb{T} ; \mathbb{R})\right) \cap B V_{\mathrm{loc}}\left(\mathbb{R}^{+} ; L_{o}^{2}(\mathbb{T} ; \mathbb{R})\right) \cap L_{\mathrm{loc}}^{\infty}\left(\mathbb{R}^{+} ; W_{o}^{1,2}(\mathbb{T} ; \mathbb{R})\right) .
$$

The assumptions above are unnecessarily strong for the claim of Lemma 2 but are needed for the crucial estimates which are obtained in the following result.

Lemma 3. Let the assumptions of Lemma 2 hold and assume in addition that there exists a point $w_{\diamond} \in \mathbb{R}$ such that $\sigma^{\prime}$ is nonincreasing on $\left(-\infty, w_{\diamond}\right)$ and nondecreasing on $\left(w_{\diamond}, \infty\right)$. Then the solution $\left(w^{\epsilon}, v^{\epsilon}\right)$ of $(8)$ that exists according to Lemma 2 satisfies for almost every $t \geq 0$ the inequalities

$$
\begin{aligned}
& \underset{x \in[-1,1]}{\operatorname{ess} \sup _{1}}\left|v^{\epsilon}(t, x)\right| \leq B^{\epsilon}(t), \\
& \underset{x \in[-1,1]}{\operatorname{ess} \sup }\left|\int_{w_{\diamond}}^{w^{\epsilon}(t, x)} \sqrt{\sigma^{\prime}(s)} d s\right| \leq 2 B^{\epsilon}(t) \text {, }
\end{aligned}
$$

where

$$
\begin{aligned}
B^{\epsilon}(t)= & \int_{0}^{t} \underset{x \in[-1,1]}{\operatorname{ess} \sup _{t}}\left|g_{t}^{\epsilon}(s, x)\right| d s+\underset{x \in[-1,1]}{\operatorname{ess} \sup }\left|g^{\epsilon}(0, x)\right| \\
& +\underset{x \in[-1,1]}{\operatorname{ess} \sup _{1}}\left|\int_{w_{\diamond}}^{w_{0}^{\epsilon}(x)} \sqrt{\sigma^{\prime}(s)} d s\right|, \quad t \geq 0,
\end{aligned}
$$

and for each $T>0$ there is a constant $C$ that depends on $T,\left\|w_{0}^{\epsilon}\right\|_{L^{\infty}(\mathbb{T})}$, $\left\|g_{t}^{\epsilon}\right\|_{L^{2}\left([0, T] ; L^{2}(\mathbb{T})\right)}$, and $\left\|g^{\epsilon}(0, \bullet)\right\|_{L^{\infty}(\mathbb{T})}$ only, such that

$$
\epsilon \int_{0}^{T} \int_{-1}^{1}\left(\left|w_{x}^{\epsilon}(t, x)\right|^{2}+\left|v_{x}^{\epsilon}(t, x)\right|^{2}\right) d x d t \leq C,
$$

and

$$
\begin{aligned}
\int_{0}^{T} \int_{0}^{t} \int_{-1}^{1}\left(\left|w^{\epsilon}(t-s, x)-w^{\epsilon}(t, x)\right|^{2}\right. \\
\left.+\left|v^{\epsilon}(t-s, x)-v^{\epsilon}(t, x)\right|^{2}\right) d x \beta(d s) d t \leq C .
\end{aligned}
$$

We could now prove that there is a weak solution of $(6)$ but we only state the result about the weak solution of (1), and we have the following result. 
Theorem 4. Assume that

(v) $k \in L_{\mathrm{loc}}^{1}\left(\mathbb{R}^{+} ; \mathbb{R}\right)$ is positive and nonincreasing on $(0, \infty)$ and $\log (k)$ is convex on this interval;

(vi) $\sigma \in C^{1}(\mathbb{R} ; \mathbb{R})$ and there exists a point $w_{\diamond} \in \mathbb{R}$ such that $\sigma^{\prime}$ is nonincreasing on $\left(-\infty, w_{\diamond}\right)$, nondecreasing on $\left(w_{\diamond}, \infty\right)$, and $\sigma^{\prime}\left(w_{\diamond}\right)>0$;

(vii) If $\left|k^{\prime}(0+)\right|<\infty$, then the point $w_{\diamond}$ in (vi) is unique, $\sigma \in C^{2}(\mathbb{R} ; \mathbb{R})$, and $\left(w-w_{\diamond}\right) \sigma^{\prime \prime}(w)>0$ on the interval $\left(w_{\diamond}, w_{\diamond}+\delta\right)$ or on the interval $\left(w_{\diamond}-\delta, w_{\diamond}\right)$ for some $\delta>0$;

(viii) $f \in W_{\mathrm{loc}}^{1,1}\left(\mathbb{R}^{+} ; L^{\infty}([0,1] ; \mathbb{R})\right) \cap W_{\mathrm{loc}}^{1,2}\left(\mathbb{R}^{+} ; L^{2}([0,1] ; \mathbb{R})\right)$;

(ix) $u_{0} \in W_{0}^{1, \infty}([0,1] ; \mathbb{R})$.

Then there exists a function $u \in L_{\mathrm{loc}}^{\infty}\left(\mathbb{R}^{+} ; W_{0}^{1, \infty}([0,1] ; \mathbb{R})\right)$ such that $(1)$ holds in the following weak sense: For each smooth function $\varphi \in C^{\infty}\left(\mathbb{R}^{2} ; \mathbb{R}\right)$ with compact support contained in $\mathbb{R} \times(0,1)$ we have

$$
\begin{aligned}
\int_{0}^{\infty} & \int_{0}^{1} u(t, x) \varphi_{t}(t, x) d x d t+\int_{0}^{1} u_{0}(x) \varphi(0, x) d x \\
= & \int_{0}^{\infty} \int_{0}^{t} k(t-s) \int_{0}^{1} \sigma\left(u_{x}(s, x)\right) \varphi_{x}(t, x) d x d s d t \\
& -\int_{0}^{\infty} \int_{0}^{1} f(t, x) \varphi(t, x) d x d t .
\end{aligned}
$$

Moreover, there is a function $v \in L_{\mathrm{loc}}^{\infty}\left(\mathbb{R}^{+} ; L^{\infty}([0,1] ; \mathbb{R})\right)$ so that

$$
u(t, x)=u_{0}(x)+\int_{0}^{t} c(t-s) v(s, x) d s, \quad t \geq 0, x \in[0,1],
$$

in particular, $u \in W_{\mathrm{loc}}^{1, \infty}\left(\mathbb{R}^{+} ; L^{\infty}([0,1] ; \mathbb{R})\right)$ if $k(0+)<\infty$, and the following inequalities hold for almost every $t \geq 0$ :

$$
\begin{aligned}
& \underset{x \in[0,1]}{\operatorname{ess} \sup }\left|\int_{w_{o}}^{u_{x}(t, x)} \sqrt{\sigma^{\prime}(s)} d s\right| \leq 2 B(t), \\
& \underset{x \in[0,1]}{\operatorname{ess} \sup }\left|u_{t}(t, x)\right| \leq 2 \sqrt{k(0+)} B(t), \\
& \underset{x \in[0,1]}{\operatorname{ess} \sup }|v(t, x)| \leq B(t),
\end{aligned}
$$

where

$$
\begin{aligned}
B(t)= & \int_{0}^{t} \operatorname{esssup}_{x \in[0,1]}\left|g_{t}(s, x)\right| d s+\underset{x \in[0,1]}{\operatorname{ess} \sup }|g(0, x)| \\
& +\underset{x \in[0,1]}{\operatorname{ess} \sup }\left|\int_{w_{\diamond}}^{u_{0_{x}}(x)} \sqrt{\sigma^{\prime}(s)} d s\right|,
\end{aligned}
$$

and $g$ is given in (7).

The function $u$ is obtained as the limit of a sequence

$$
u_{0}(x)+\int_{0}^{t} c(t-s) v^{\epsilon_{j}}(s, x) d s
$$


when $\epsilon_{j} \downarrow 0$ where $\left(w^{\epsilon_{j}}, v^{\epsilon_{j}}\right)$ is a solution of $(8)$ with $\epsilon=\epsilon_{j}$ and the sequence $\left\{v^{\epsilon_{j}}\right\}$ converges in $L_{\text {loc }}^{2}\left(\mathbb{R}^{+} ; L^{2}([0,1] ; \mathbb{R})\right)$ when $|k(0+)|=\infty$.

We will need the following lemma that is essentially [5, Lemma A2]. The proof in [5] is quite abstract (and I cannot follow the arguments in all details) and does not cover exactly the case that we for technical reasons need here, so a proof will be given below.

Lemma 5. Let $X$ be a Hilbert space with norm $\|\bullet\|$, let $T>0$, and assume that $\alpha$ is a nonnegative measure on $[0, T]$ such that $\alpha([\epsilon, T])<\infty$ for all $\epsilon>0$ but $\alpha((0, T])=\infty$, and $\alpha$ is such that if $\varphi$ is a smooth function with support in $(0, T)$ and $\alpha_{\varphi}(E)=\int_{E \cap(0, T)} \varphi(t) \alpha(d t)$ for every Borel set $E$, then $\lim _{|\omega| \rightarrow \infty} \widetilde{\alpha_{\varphi}}(\omega)=0$ (where $\sim$ denotes the Fourier transform).

Let $u \in L^{2}([0, T] ; X)$ be such that for some constant $\Gamma$

$$
\int_{0}^{T} \int_{0}^{t}\|u(t-s)-u(t)\|^{2} \alpha(d s) d t+\int_{0}^{T}\|u(t)\|^{2} d t \leq \Gamma,
$$

and for some continuous function $\rho$ on $[0, T]$ with $\rho(0)=0$ one has

$$
\int_{0}^{h}\|u(t)\|^{2} d t \leq \rho(h), \quad h \in[0, T] .
$$

Then there exists a continuous function $\eta$ on $[0, T]$ with $\eta(0)=0$, which depends on $\alpha, \Gamma$, and $\rho$ only, such that

$$
\int_{0}^{T-\tau}\|u(t+\tau)-u(t)\|^{2} d t \leq \eta(\tau), \quad \tau \in[0, T] .
$$

It is clear that if $\alpha(E)=\int_{E} A(s) d s$ for some nonnegative measurable function $A$, then the assumption on the Fourier transform is automatically satisfied, and in this case $X$ can be a Banach space.

\section{PROOFS}

Proof of Lemma 1. The statements about $a$ follow essentially from [8, Theorem 3] since the case where $k(0+)<\infty$ can be handled in the same manner as the one where $k(0+)=\infty$. Concerning $b$ we see that the Laplace transform (denoted by “" ") of $\gamma \delta+b$ must be $1 / \sqrt{\bullet \hat{k}(\bullet)}$. Following [28] we use a standard result from complex analysis to get

$$
\begin{aligned}
\frac{1}{\sqrt{z \hat{k}(z)}} & =\frac{1}{\pi} \int_{0}^{\infty} \frac{1}{(\lambda+z \hat{k}(z)) \sqrt{\lambda}} d \lambda \\
& =\frac{1}{\pi} \int_{0}^{\infty} \frac{1}{z(\lambda / z+\hat{k}(z)) \sqrt{\lambda}} d \lambda, \quad \Re z>0 .
\end{aligned}
$$

Since $k$ is assumed to be nonincreasing and log-convex, it follows that the function $\lambda+k(\bullet)$ is nonincreasing and log-convex as well. Hence there exists (again by [8, Theorem 3]) a nonnegative and nonincreasing solution $b_{\lambda}$ of the equation

$$
\frac{1}{\lambda+k(0+)}(\lambda+k(t))+\int_{0}^{t} b_{\lambda}(t-s)(\lambda+k(s)) d s=1, \quad t>0
$$


for each $\lambda>0$, and it is clear that

$$
\int_{0}^{t} b_{\lambda}(s) d s \leq \frac{1}{\lambda+k(t)}, \quad t>0 .
$$

Thus we easily see from (19) that $b$ is given by

$$
b=\frac{1}{\pi} \int_{0}^{\infty} b_{\lambda}(\bullet) \frac{1}{\sqrt{\lambda}} d \lambda,
$$

and it follows that $b$ is nonnegative and nonincreasing as well. If $k(0+)=$ $\infty$ it follows directly from (5) that $b(0+)=\infty$ because otherwise one would have $a(0+)=0$. If $k(0+)<\infty$ but $\left|k^{\prime}(0+)\right|=\infty$ it follows from (3) by differentiating both sides that we have $a(0+)=\infty$ and thus we again get that $b(0+)=\infty$ from $(5)$.

If $\gamma>0$ it is clear that there is a solution $c$ of (9) and if $\gamma=0$ one can use [8, Theorem 1] to show that a solution $c$ exists, but in this case one must show that $c$ is a function and not a measure. We do this by first showing that if $\gamma>0$, so that $k(0+)<\infty$, then $c$ must be nonincreasing, and having done this we can invoke a straightforward approximation argument (e.g., replace $k$ by $k(\bullet+1 / n))$ to show that $c$ must be nonincreasing in the case where $\gamma=0$ as well.

Suppose that $k(0+)<\infty$ so that $\gamma>0$. Equation (10) is an immediate consequence of the fact that $\hat{c}(z)=\sqrt{\hat{k}(z) / z}$, and from (9) we see that $c \in$ $A C_{\text {loc }}\left(\mathbb{R}^{+} ; \mathbb{R}\right)$. If we differentiate both sides of $(10)$ twice we get

$$
2 c(0) c^{\prime}(t)=k^{\prime}(t)-\int_{0}^{t} c^{\prime}(t-s) c^{\prime}(s) d s, \quad t>0,
$$

and because $c(0)=1 / \gamma>0$ and $k^{\prime}$ is nonpositive we see that $c$ must be nonincreasing. Since it is now easy to see that $c$ must be nonnegative, we have obtained the desired result.

Proof of Lemma 2. In order to show that there exists a solution of (8) we invoke $\left[10\right.$, Theorem 4] where $X$ is the Hilbert space $L_{e}^{2}(\mathbb{T} ; \mathbb{R}) \times L_{o}^{2}(\mathbb{T} ; \mathbb{R})$, the operator $A$ is defined by

$$
A\left(\begin{array}{c}
w \\
v
\end{array}\right)=\left(\begin{array}{c}
-\epsilon w_{x x}-v_{x}+\kappa w \\
-\epsilon v_{x x}-(\sigma(w))_{x}+\kappa v
\end{array}\right)
$$

with $D(A)=W_{e}^{2,2}(\mathbb{T} ; \mathbb{R}) \times W_{o}^{2,2}(\mathbb{T} ; \mathbb{R})$, and

$$
G\left(\begin{array}{c}
w \\
v
\end{array}\right)(t)=\left(\begin{array}{c}
\kappa w(t, \bullet) \\
\kappa v(t, \bullet)+\gamma g_{t}^{\epsilon}(t, \bullet)+\int_{0}^{t} b(t-s) g_{t}^{\epsilon}(s, \bullet) d s
\end{array}\right) .
$$

It is straightforward to check that if $\kappa$ is chosen to be sufficiently large (depending on $\epsilon$ ) then $A$ is $m$-accretive, i.e., maximal monotone, because $\sigma$ is Lipschitz-continuous and for the the study of the linear part one can use Fourier series (a cosine series for the even component and a sine series for the odd component). Thus we get the existence of a strong solution $\left(w^{\epsilon}, v^{\epsilon}\right)$ from [10, Theorem 4]. We must, however, establish some additional regularity properties of the solution as well, in particular we must show that $w^{\epsilon}$ and $v^{\epsilon}$ belong to $L_{\text {loc }}^{\infty}\left(\mathbb{R}^{+} ; L^{\infty}(\mathbb{T} ; \mathbb{R})\right)$. To do this we define the operator $A_{0}$ by

$$
A_{0}\left(\begin{array}{c}
w \\
v
\end{array}\right)=\left(\begin{array}{l}
-\epsilon w_{x x} \\
-\epsilon v_{x x}
\end{array}\right)
$$


with $D\left(A_{0}\right)=D(A)$ and note that $A_{0}$ is the subdifferential of the convex function $\mathbf{u} \mapsto \varphi(\mathbf{u})=2^{-1} \epsilon \int_{-1}^{1}\left(\left|w_{x}(x)\right|^{2}+\left|v_{x}(x)\right|^{2}\right) d x$ where $\mathbf{u}=(w, v)$. By definition this means that $\varphi\left(\mathbf{u}_{1}\right)-\varphi\left(\mathbf{u}_{2}\right) \geq\left\langle A_{0}\left(\mathbf{u}_{2}\right), \mathbf{u}_{1}-\mathbf{u}_{2}\right\rangle_{X}$. Another important point is that

$$
\left\langle A_{0}(\mathbf{u}), A(\mathbf{u})\right\rangle_{X} \geq\left\|A_{0}(\mathbf{u})\right\|_{X}^{2} / 2,
$$

if $\kappa$ is large enough. In the notation of [10] we have the approximating equation

$$
L_{\lambda}\left(\mathbf{u}_{\lambda}(\bullet)-\mathbf{u}_{\lambda}(0)\right)(t)+A\left(\mathbf{u}_{\lambda}(t)\right)=G\left(\mathbf{u}_{\lambda}\right)(t), \quad t \geq 0, \lambda>0,
$$

where $L_{\lambda}(\psi)=\lambda^{-1}\left(\psi(t)-\int_{0}^{t} r_{\lambda}(s) \psi(t-s) d s\right)$ and $\lambda r_{\lambda}(t)+\int_{0}^{t} r_{\lambda}(t-s) c(s) d s=$ $c(t)$. In particular, the fact that $b$ is nonincreasing implies that $r_{\lambda}$ is nonnegative. This in turn implies since $A_{0}$ is the subdifferential of $\varphi$ that

$$
\begin{aligned}
&\left\langle A_{0}\left(\mathbf{u}_{\lambda}(t)\right), L_{\lambda}\left(\mathbf{u}_{\lambda}(\bullet)-\mathbf{u}_{\lambda}(0)\right)(t)\right\rangle_{X} \\
&=\frac{1}{\lambda}\left(1-\int_{0}^{r} r_{\lambda}(s) d s\right)\left\langle A_{0}\left(\mathbf{u}_{\lambda}(t)\right), \mathbf{u}_{\lambda}(t)-\mathbf{u}_{\lambda}(0)\right\rangle_{X} \\
& \quad+\frac{1}{\lambda} \int_{0}^{t} r_{\lambda}(s)\left\langle A_{0}\left(\mathbf{u}_{\lambda}(t)\right), \mathbf{u}_{\lambda}(t)-\mathbf{u}_{\lambda}(t-s)\right\rangle_{X} d s \\
& \geq \frac{1}{\lambda}\left(1-\int_{0}^{r} r_{\lambda}(s) d s\right)\left(\varphi\left(\mathbf{u}_{\lambda}(t)\right)-\varphi\left(\mathbf{u}_{\lambda}(0)\right)\right) \\
& \quad+\frac{1}{\lambda} \int_{0}^{t} r_{\lambda}(s)\left(\varphi\left(\mathbf{u}_{\lambda}(t)\right)-\varphi\left(\mathbf{u}_{\lambda}(t-s)\right)\right) d s \\
&= L_{\lambda}\left(\varphi\left(\mathbf{u}_{\lambda}(\bullet)\right)-\varphi\left(\mathbf{u}_{\lambda}(0)\right)\right) .
\end{aligned}
$$

Since $L_{\lambda}^{-1}(\psi)(t)=\lambda \psi(t)+\int_{0}^{t} c(t-s) \psi(s) d s$ and $c$ is nonnegative, we can deduce from the preceding inequality combined with (20) and (21) that $\varphi\left(\mathbf{u}_{\lambda}(t)\right)$ is uniformly bounded for $t$ in a compact interval $[0, T]$ and $\lambda \in(0,1)$. But then this result holds for the limit function $\mathbf{u}^{\epsilon}$ as well, and this was what we had to prove.

Proof of Lemma 3. Recall that a continuously differentiable function $\eta: \mathbb{R}^{2} \rightarrow \mathbb{R}$ is an entropy for the conservation law

$$
w_{t}(t, x)=v_{x}(t, x), \quad v_{t}(t, x)=(\sigma(w))_{x}(t, x),
$$

with corresponding entropy flux $q$ if

$$
\frac{\partial}{\partial t} \eta(w(t, x), v(t, x))+\frac{\partial}{\partial x} q(w(t, x), v(t, x))=0
$$

for every smooth solution $(w, v)$ of $(22)$. Such pairs $(\eta, q)$ are obtained as solutions of the system of equations

$$
q_{w}(w, v)=-\sigma^{\prime}(w) \eta_{v}(w, v), \quad q_{v}(w, v)=-\eta_{w}(w, v)
$$

Thus it is easy to see that in order to be able to find a solution of this system it suffices to find a function $\eta$ that satisfies

$$
\eta_{w w}(w, v)=\sigma^{\prime}(w) \eta_{v v}(w, v), \quad(w, v) \in \mathbb{R}^{2}
$$

Now the main idea in this proof is to choose a suitable entropy function $\eta$ and multiply both sides of the equations in (8) by $\eta_{w}\left(w^{\epsilon}(t, x), v^{\epsilon}(t, x)\right)$ and 
$\eta_{v}\left(w^{\epsilon}(t, x), v^{\epsilon}(t, x)\right)$, respectively, add the results and integrate both sides of the equality so obtained over $[-1,1]$. We have to consider the resulting terms separately.

We use the notation

$$
\mathbf{u}^{\epsilon}(t, x)=\left(w^{\epsilon}(t, x), v^{\epsilon}(t, x)\right), \quad \mathbf{u}_{0}^{\epsilon}(x)=\left(w_{0}^{\epsilon}(x), g^{\epsilon}(0, x)\right),
$$

denote by $\partial \eta$ the gradient $\left(\eta_{w}\left(w^{\epsilon}, v^{\epsilon}\right), \eta_{v}\left(w^{\epsilon}, v^{\epsilon}\right)\right)$ of $\eta$, and by $\langle\bullet, \bullet\rangle$ the standard inner product in $\mathbb{R}^{2}$. Furthermore, we recall that if $\psi$ is a function that is locally of bounded variation on $\mathbb{R}^{+}$, then

$\frac{\partial}{\partial t} \int_{0}^{t} b(t-s)(\psi(s)-\psi(0)) d s=b(t)(\psi(t)-\psi(0))-\int_{(0, t)}(\psi(t-s)-\psi(t)) \beta(d s)$, and we shall apply this result to the functions $t \mapsto w^{\epsilon}(t, \bullet)$ and $t \mapsto v^{\epsilon}(t, \bullet)$.

Thus it follows from the convexity of the function $\eta$ and the fact that $\beta$ is nonnegative that

$$
\begin{aligned}
\eta_{w}\left(\mathbf{u}^{\epsilon}(t, x)\right) \frac{\partial}{\partial t}\left(\gamma\left(w^{\epsilon}(t, x)-w_{0}^{\epsilon}(x)\right)\right. & \left.+\int_{0}^{t} b(t-s)\left(w^{\epsilon}(s, x)-w_{0}^{\epsilon}(x)\right) d s\right) \\
& +\eta_{v}\left(\mathbf{u}^{\epsilon}(t, x)\right) \frac{\partial}{\partial t}\left(\gamma\left(v^{\epsilon}(t, x)-g^{\epsilon}(0, x)\right)\right. \\
& \left.\quad+\int_{0}^{t} b(t-s)\left(v^{\epsilon}(s, x)-g^{\epsilon}(0, x)\right) d s\right) \\
= & \gamma \frac{\partial}{\partial t} \eta\left(\mathbf{u}^{\epsilon}(t, x)\right)+b(t)\left(\eta\left(\mathbf{u}^{\epsilon}(t, x)\right)-\eta\left(\mathbf{u}_{0}^{\epsilon}(x)\right)\right) \\
& -\int_{(0, t)}\left(\eta\left(\mathbf{u}^{\epsilon}(t-s, x)\right)-\eta\left(\mathbf{u}^{\epsilon}(t, x)\right)\right) \beta(d s) \\
& +b(t)\left(\eta\left(\mathbf{u}_{0}^{\epsilon}(x)\right)-\eta\left(\mathbf{u}^{\epsilon}(t, x)\right)-\left\langle\partial \eta\left(\mathbf{u}^{\epsilon}(t, x)\right), \mathbf{u}_{0}^{\epsilon}(x)-\mathbf{u}^{\epsilon}(t, x)\right\rangle\right) \\
& +\int_{(0, t)}\left(\eta\left(\mathbf{u}^{\epsilon}(t-s, x)\right)-\eta\left(\mathbf{u}^{\epsilon}(t, x)\right)\right. \\
\left.\quad-\left\langle\partial \eta\left(\mathbf{u}^{\epsilon}(t, x)\right), \mathbf{u}^{\epsilon}(t-s, x)-\mathbf{u}^{\epsilon}(t, x)\right\rangle\right) \beta(d s) & \\
\geq & \frac{\partial}{\partial t}\left(\gamma\left(\eta\left(\mathbf{u}^{\epsilon}(t, x)\right)-\eta\left(\mathbf{u}_{0}^{\epsilon}(x)\right)\right)\right. \\
& \left.+\int_{0}^{t} b(t-s)\left(\eta\left(\mathbf{u}^{\epsilon}(s, x)\right)-\eta\left(\mathbf{u}_{0}^{\epsilon}(x)\right)\right) d s\right) .
\end{aligned}
$$

Because $\eta$ is an entropy there is an entropy flux $q$ satisfying (23) and therefore we get

$$
\begin{gathered}
\int_{-1}^{1}\left(\eta_{w}\left(\mathbf{u}^{\epsilon}(t, x)\right) v_{x}^{\epsilon}(t, x)+\eta_{v}\left(\mathbf{u}^{\epsilon}(t, x)\right) \sigma^{\prime}\left(w^{\epsilon}(t, x)\right) w_{x}^{\epsilon}(t, x)\right) d x \\
\quad=\int_{-1}^{1}\left(-q_{v}\left(\mathbf{u}^{\epsilon}(t, x)\right) v_{x}^{\epsilon}(t, x)-q_{w}\left(\mathbf{u}^{\epsilon}(t, x)\right) w_{x}^{\epsilon}(t, x)\right) d x \\
=-\int_{-1}^{1} \frac{\partial}{\partial x} q\left(\mathbf{u}^{\epsilon}(t, x)\right) d x=0,
\end{gathered}
$$

by the periodicity. 
Moreover, by the convexity of $\eta$ we have (27)

$$
\begin{aligned}
\int_{-1}^{1}( & \left.\eta_{w}\left(\mathbf{u}^{\epsilon}(t, x)\right) w_{x x}^{\epsilon}(t, x)+\eta_{v}\left(\mathbf{u}^{\epsilon}(t, x)\right) v_{x x}^{\epsilon}(t, x)\right) d x \\
= & \int_{-1}^{1}\left(\frac{\partial}{\partial x}\left(\frac{\partial}{\partial x} \eta\left(\mathbf{u}^{\epsilon}(t, x)\right)\right)\right) d x \\
& -\int_{-1}^{1}\left(\eta_{w w}\left(\mathbf{u}^{\epsilon}(t, x)\right) w_{x}^{\epsilon}(t, x)^{2}+\eta_{v v}\left(\mathbf{u}^{\epsilon}(t, x)\right) v_{x}^{\epsilon}(t, x)^{2}\right. \\
& \left.+2 \eta_{w v}\left(\mathbf{u}^{\epsilon}(t, x)\right) w_{x}^{\epsilon}(t, x) v_{x}^{\epsilon}(t, x)\right) d x \\
= & -\int_{-1}^{1}\left(\eta_{w w}\left(\mathbf{u}^{\epsilon}(t, x)\right) w_{x}^{\epsilon}(t, x)^{2}+\eta_{v v}\left(\mathbf{u}^{\epsilon}(t, x)\right) v_{x}^{\epsilon}(t, x)^{2}\right. \\
+ & \left.2 \eta_{w v}\left(\mathbf{u}^{\epsilon}(t, x)\right) w_{x}^{\epsilon}(t, x) v_{x}^{\epsilon}(t, x)\right) d x \leq 0 .
\end{aligned}
$$

First let us choose the entropy to be

$$
\eta(w, v)=\int_{0}^{w} \sigma(s) d s+\frac{1}{2} v^{2}
$$

in which case we have $q(w, v)=-v \sigma(w)$. (If $\sigma(0)=0$, then $\eta$ is nonnegative, but in any case we have $\inf _{w, v \in \mathbb{R}} \eta(w, v)>-\infty$.) We define the functions $\xi$ and $h$ by

$$
\begin{aligned}
\xi(t) & =\int_{-1}^{1}\left(\eta\left(\mathbf{u}^{\epsilon}(t, x)\right)-\eta\left(\mathbf{u}_{0}^{\epsilon}(x)\right)\right) d x \\
h(t, x) & =\frac{\partial}{\partial t}\left(\gamma(g(t, x)-g(0, x))+\int_{0}^{t} b(t-s)(g(t, x)-g(0, x)) d s\right),
\end{aligned}
$$

where $t \geq 0$ and $x \in \mathbb{T}$. Now we deduce from (8) and (25)-(27) that (28)

$$
\begin{gathered}
\frac{\partial}{\partial t}\left(\gamma \xi(t)+\int_{0}^{t} b(t-s) \xi(s) d s\right)+\epsilon \int_{-1}^{1}\left(\sigma^{\prime}\left(w^{\epsilon}(t, x)\right) w_{x}^{\epsilon}(t, x)^{2}+v_{x}^{\epsilon}(t, x)^{2}\right) d x \\
\leq \int_{-1}^{1} v^{\epsilon}(t, x) h(t, x) d x \leq \int_{-1}^{1} \eta\left(\mathbf{u}^{\epsilon}(t, x)\right) d x+\frac{1}{2} \int_{-1}^{1}|h(t, x)|^{2} d x
\end{gathered}
$$

Next we recall that the second term on the left-hand side is nonnegative and therefore we get, when we convolve both sides of the inequality with the nonnegative function $c$ and use the fact that $\xi(0)=0$ that

$$
\xi(t) \leq \int_{0}^{t} c(t-s)\left(\int_{-1}^{1} \eta\left(\mathbf{u}^{\epsilon}(t, x)\right) d x+\frac{1}{2} \int_{-1}^{1}|h(t, x)|^{2} d x\right),
$$

or equivalently

$$
\begin{aligned}
\int_{-1}^{1} \eta\left(\mathbf{u}^{\epsilon}(t, x)\right) d x \leq & \int_{-1}^{1} \eta\left(\mathbf{u}^{\epsilon}(0, x)\right) d x \\
& +\int_{0}^{t} c(t-s)\left(\int_{-1}^{1} \eta\left(\mathbf{u}^{\epsilon}(t, x)\right) d x+\frac{1}{2} \int_{-1}^{1}|h(t, x)|^{2} d x\right) .
\end{aligned}
$$


It follows from this inequality by standard results for Volterra equations that $\int_{0}^{T} \int_{-1}^{1} \eta\left(\mathbf{u}^{\epsilon}(t, x)\right) d x d t$ is, for each $T>0$, bounded by a constant that depends on $T, k, \sigma,\left\|g_{t}^{\epsilon}\right\|_{L^{2}\left([0, T] ; L^{2}(\mathbb{T})\right)},\left\|w_{0}^{\epsilon}\right\|_{L^{\infty}(\mathbb{T})}$, and $\left\|g^{\epsilon}(0, \bullet)\right\|_{L^{\infty}(\mathbb{T})}$ only. But this means that if we integrate both sides of $(28)$ over $(0, T)$, then we get (13), because $\inf _{s \in \mathbb{R}} \sigma^{\prime}(s)>0$.

In the same way we conclude from (25) that for each $T$

$$
\begin{aligned}
\int_{0}^{T} \int_{(0, t)}\left(\eta\left(\mathbf{u}^{\epsilon}(t-s, x)\right)-\eta\left(\mathbf{u}^{\epsilon}(t, x)\right)\right. \\
\left.-\left\langle\partial \eta\left(\mathbf{u}^{\epsilon}(t, x)\right), \mathbf{u}^{\epsilon}(t-s, x)-\mathbf{u}^{\epsilon}(t, x)\right\rangle\right) \beta(d s) d t \leq C,
\end{aligned}
$$

where $C$ is again a constant that depends on $T, k, \sigma,\left\|g_{t}^{\epsilon}\right\|_{L^{2}\left([0, T] ; L^{2}(\mathbb{T})\right)}$, $\left\|w_{0}^{\epsilon}\right\|_{L^{\infty}(\mathbb{T})}$, and $\left\|g^{\epsilon}(0, \bullet)\right\|_{L^{\infty}(\mathbb{T})}$ only. Thus we get (14) because

$$
\int_{w_{1}}^{w_{2}} \sigma(s) d s-\sigma\left(w_{1}\right)\left(w_{2}-w_{1}\right) \geq \frac{1}{2} \inf _{s \in \mathbb{R}} \sigma^{\prime}(s)\left|w_{2}-w_{1}\right|^{2},
$$

and

$$
\frac{1}{2}\left(v_{2}^{2}-v_{1}^{2}-2 v_{1}\left(v_{2}-v_{1}\right)\right)=\frac{1}{2}\left|v_{2}-v_{1}\right|^{2} .
$$

Next we consider the following standard family of entropies. Let $k$ be an arbitrary positive number (that we eventually have to choose to be arbitrarily large) and let

$$
\eta_{k}(w, v)=e^{k \int_{w_{\diamond}}^{w} \psi_{k}(s) d s+k v},
$$

in which case the corresponding entropy fluxes are

$$
q_{k}(w, v)=-\psi_{k}(w) \eta_{k}(w, v) .
$$

In order for (23) to be satisfied, the function $\psi_{k}$ must be a solution of the differential equation

$$
\psi_{k}^{\prime}(w)+k \psi_{k}^{2}(w)=k \sigma^{\prime}(w) .
$$

We require that $\psi_{k}$ satisfy the initial condition $\psi_{k}\left(w_{\diamond}\right)=0$. The assumptions on $\sigma$ will then guarantee that $\psi_{k}$ is nondecreasing and this implies in turn that the function $\eta_{k}$ is convex, as one can easily check. Moreover, it is easy to prove with the aid of $(29)$ that

$$
\lim _{k \rightarrow \infty} \int_{w_{\diamond}}^{w} \psi_{k}(s) d s=\left|\int_{w_{\diamond}}^{w} \sqrt{\sigma^{\prime}(s)} d s\right|, \quad w \in \mathbb{R} .
$$

Let

$$
G_{+}^{\epsilon}(t)=\int_{0}^{t} \operatorname{esssup}_{x \in[-1,1]} g_{t}^{\epsilon}(s, x) d s, \quad t \geq 0 .
$$

In (8) we can now replace $v^{\epsilon}$ by the function $v_{+}^{\epsilon}(t, x)=v^{\epsilon}(t, x)-G_{+}^{\epsilon}(t)$ provided we replace $g^{\epsilon}$ by the function $g_{+}^{\epsilon}(t, x)=g^{\epsilon}(t, x)-G_{+}^{\epsilon}(t)$, because the function $G_{+}^{\epsilon}$ does not depend on $x$ and therefore the terms $v_{x}^{\epsilon}$ and $\epsilon v_{x x}^{\epsilon}$ are not affected by this change. Note that the reason for doing this is that now $g_{+}^{\epsilon}(t, x)$ is for almost every $x$ a nonincreasing function and it follows in particular that for almost every $x \in[-1,1]$ and $t \geq 0$ one has

$$
\frac{\partial}{\partial t}\left(\gamma g_{+}^{\epsilon}(t, x)+\int_{0}^{t} b(t-s) g_{+}^{\epsilon}(t, x) d s\right) \leq 0 .
$$


If we combine this result with the fact that the entropy $\eta_{k}$ is now increasing in its second argument, i.e., $\eta_{k v}(w, v)>0$, we conclude from (25), (26), and (27) that

$$
\int_{-1}^{1} \eta_{k}\left(\mathbf{u}^{\epsilon}(t, x)\right) d x \leq \int_{-1}^{1} \eta_{k}\left(\mathbf{u}_{0}^{\epsilon}(x)\right) d x
$$

where $t \geq 0$. Taking the $k$ th root of both sides of this equation and letting $k \rightarrow \infty$ we deduce with the aid of (30) that

$$
\begin{array}{r}
\underset{x \in[-1,1]}{\operatorname{ess} \sup _{1}}\left(\left|\int_{w_{\diamond}}^{w(t, x)} \sqrt{\sigma^{\prime}(s)} d s\right|+v(t, x)-G_{+}^{\epsilon}(t)\right) \\
\quad \leq \operatorname{esssup}_{x \in[-1,1]}\left(\left|\int_{w_{\diamond}}^{w(0, x)} \sqrt{\sigma^{\prime}(s)} d s\right|+v(0, x)\right) .
\end{array}
$$

Thus we conclude that

$$
\begin{aligned}
\underset{x \in[-1,1]}{\operatorname{ess} \sup } v(t, x) \leq & \int_{0}^{t} \operatorname{esssup}_{x \in[-1,1]} g_{t}(s, x) d s \\
& +\operatorname{esssup}_{x \in[-1,1]}\left(\left|\int_{w_{0}}^{w(0, x)} \sqrt{\sigma^{\prime}(s)} d s\right|+v(0, x)\right) .
\end{aligned}
$$

In the same way, by choosing as entropy the function $e^{k \int_{w_{\odot}}^{w} \psi_{k}(s) d s-k v}$, we can get a lower bound of the form

$$
\begin{aligned}
\underset{x \in[-1,1]}{\operatorname{essinf}} v(t, x) \geq & \int_{0}^{t} \underset{x \in[-1,1]}{\operatorname{essinf}} g_{t}(s, x) d s \\
& +\underset{x \in[-1,1]}{\operatorname{essinf}}\left(-\left|\int_{w_{0}}^{w(0, x)} \sqrt{\sigma^{\prime}(s)} d s\right|+v(0, x)\right) .
\end{aligned}
$$

Combining (32) and (33) we get the first part of (11). Finally, if we insert (33) in (31) then we get the following upper bound for the function $w$;

$$
\begin{aligned}
& \underset{x \in[-1,1]}{\operatorname{ess} \sup _{1}}\left|\int_{w_{\diamond}}^{w(t, x)} \sqrt{\sigma^{\prime}(s)} d s\right| \leq 2 \underset{x \in[-1,1]}{\operatorname{ess} \sup }\left|\int_{w_{\diamond}}^{w(0, x)} \sqrt{\sigma^{\prime}(s)} d s\right| \\
& \quad+2 \underset{x \in[-1,1]}{\operatorname{ess} \sup }|v(0, x)|+2 \int_{0}^{t} \operatorname{esssup}_{x \in[-1,1]}\left|g_{t}(s, x)\right| d s,
\end{aligned}
$$

and thus we have obtained the second part of (11).

Proof of Lemma 5. It follows from the assumptions that for each $h \in(0, T]$ there exists a nonnegative smooth function $\varphi_{h}$ with support contained in $(0, h)$ such that $0 \leq \varphi_{h}(t) \leq 1, t \in \mathbb{R}$, but

$$
h \int_{(0, h)} \varphi_{h}(s) \alpha(d s)=1 .
$$

Define the measure $\alpha_{h}$ by

$$
\alpha_{h}=h \varphi_{h}(\bullet) \alpha(d \bullet)
$$


We pick some smooth function $e_{h}$ with support contained in $(0, T)$ such that

$$
\sup _{\omega \in \mathbb{R}}\left|\widetilde{e_{h}}(\omega)-\widetilde{\alpha_{h}}(\omega)\right| \leq h, \quad h \in(0, T] .
$$

This is possible precisely because we have $\lim _{|\omega| \rightarrow \infty} \widetilde{\alpha_{h}}(\omega)=0$ by assumption. Denote by $u_{\tau}, \tau \geq 0$, the function $u(\bullet+\tau)$ on $[0, T-\tau]$ and by $*$ the convolution product, i.e.,

$$
f * v=\int_{0}^{\bullet} f(\bullet-s) v(s) d s \quad \text { and } \quad \alpha_{h} * v=\int_{(0, \bullet)} v(\bullet-s) \alpha_{h}(d s) .
$$

By the triangle inequality we have

$$
\begin{aligned}
\left\|u_{\tau}-u\right\|_{L^{2}([0, T-\tau])} \leq & \left\|u_{\tau}-e_{h} * u_{\tau}\right\|_{L^{2}([0, T-\tau])} \\
& +\left\|u-e_{h} * u\right\|_{L^{2}([0, T-\tau])}+\left\|e_{h} *\left(u_{\tau}-u\right)\right\|_{L^{2}([0, T-\tau])} .
\end{aligned}
$$

Because $X$ is a Hilbert space the norm of the operator $v \in L^{2}(I ; X) \rightarrow f * v \in$ $L^{2}(I ; X)$ is at most $\sup _{\omega \in \mathbb{R}}|\tilde{f}(\omega)|$ (and this is exactly the norm in case $I=\mathbb{R}$ ) and we conclude that

$$
\begin{aligned}
\left\|u_{\tau}-e_{h} * u_{\tau}\right\|_{L^{2}([0, T-\tau]) \leq} \leq & \left\|u_{\tau}-\alpha_{h} * u_{\tau}\right\|_{L^{2}([0, T-\tau])} \\
& +\sup _{\omega \in \mathbb{R}} \widetilde{e_{h}}(\omega)-\widetilde{\alpha_{h}}(\omega)\|\| u_{\tau} \|_{L^{2}([0, T-\tau])} \\
\leq & \left\|u_{\tau}-\alpha_{h} * u_{\tau}\right\|_{L^{2}([0, T-\tau])}+h \sqrt{\Gamma} .
\end{aligned}
$$

We have

$$
\begin{aligned}
\| u_{\tau}- & \alpha_{h} * u_{\tau} \|_{L^{2}([0, T-\tau])}^{2} \\
= & \int_{0}^{T-\tau}\left\|\left(1-\alpha_{h}((0, t))\right) u_{\tau}(t)-\int_{(0, t)}\left(u_{\tau}(t-s)-u_{\tau}(t)\right) \alpha_{h}(d s)\right\|^{2} d t \\
\leq & 2 \int_{0}^{T-\tau}\left(1-\alpha_{h}((0, t))\right)^{2}\left\|u_{\tau}(t)\right\|^{2} d t \\
& +2 \int_{0}^{T-\tau} \int_{(0, t)}\left\|u_{\tau}(t-s)-u_{\tau}(t)\right\|^{2} \alpha_{h}(d s) d t,
\end{aligned}
$$

where we used Hölder's inequality and the fact that $\int_{(0, T)} \alpha_{h}(d s)=1$. Because

$$
\int_{0}^{T-\tau}\left(1-\int_{(0, t)} \alpha_{h}(d s)\right)^{2}\left\|u_{\tau}(t)\right\|^{2} d t \leq \int_{0}^{h}\left\|u_{\tau}(t)\right\|^{2} d t \leq \rho(h+\tau),
$$

and because we get for the second term by changing variables and using the definition of $\alpha_{h}$ that

$$
\begin{aligned}
\int_{0}^{T-\tau} & \int_{(0, t)}\left\|u_{\tau}(t-s)-u_{\tau}(t)\right\|^{2} \alpha_{h}(d s) d t \\
\quad & \int_{\tau}^{T} \int_{(0, t-\tau)}\|u(t-s)-u(t)\|^{2} \alpha_{h}(d s) d t \\
& \leq h \int_{0}^{T} \int_{(0, t)}\|u(t-s)-u(t)\|^{2} \alpha(d s) d t \leq \Gamma h,
\end{aligned}
$$


we conclude from (38) that

$$
\left\|u_{\tau}-\alpha_{h} * u_{\tau}\right\|_{L^{2}([0, T-\tau])}^{2} \leq 2 \rho(\tau+h)+2 \Gamma h .
$$

Note that this inequality holds for $\tau=0$ as well.

For every $t \in[0, T-\tau]$ we have

$$
\begin{aligned}
\int_{0}^{t} e_{h}(t-s)(u(s)-u(s+\tau)) d s \\
=\int_{0}^{t} e_{h}(t-s) u(s) d s-\int_{\tau}^{t+\tau} e_{h}(t+\tau-s) u(s) d s \\
=\int_{0}^{\tau} e_{h}(t-s) u(s) d s+\int_{\tau}^{t}\left(e_{h}(t-s)-e_{h}(t+\tau-s)\right) u(s) d s \\
\quad-\int_{t}^{t+\tau} e_{h}(t+\tau-s) u(s) d s
\end{aligned}
$$

and therefore we get, because $\left|e_{h}(t-s)-e_{h}(t+\tau-s)\right| \leq \tau \max _{t \in[0, T]}\left|e_{h}^{\prime}(t)\right|$,

$$
\begin{aligned}
\| e_{h} * & \left(u_{\tau}-u\right) \|_{L^{2}([0, T-\tau])} \\
\leq & \left\|e_{h}\right\|_{L^{1}([0, T])}\|u\|_{L^{2}([0, \tau])} \\
& +\left(\left\|e_{h}-e_{h_{\tau}}\right\|_{L^{1}([0, T-\tau])}+\left\|e_{h}\right\|_{L^{1}([0, \tau])}\right)\|u\|_{L^{2}([0, T])} \\
\leq & \left\|e_{h}\right\|_{L^{1}([0, T])} \sqrt{\rho(\tau)}+\left(\tau T \max _{t \in[0, T]}\left|e_{h}^{\prime}(t)\right|+\left\|e_{h}\right\|_{L^{1}([0, \tau])}\right) \sqrt{\Gamma} .
\end{aligned}
$$

If we combine this result with (36), (37), and (39) (with $\tau=0$ as well) we get the desired conclusion by choosing $h$ appropriately.

Proof of Theorem 4. Since we will prove that $u_{x}$ is bounded we may, without loss of generality, assume that $\sigma^{\prime}$ is bounded from above. We need this assumption if we want to apply Lemma 2 and it will not, of course, affect the bounds that we get. (For more details on this kind of argument, see [27, p. $116]$.

It is easy to see that one can find functions $g^{\epsilon}$ and $w_{0}^{\epsilon}$ that satsify (iii) and (iv) and are such that

$$
\begin{aligned}
g^{\epsilon} \rightarrow g & \text { in } W_{\text {loc }}^{1,1}\left(\mathbb{R}^{+} ; L^{\infty}(\mathbb{T} ; \mathbb{R})\right) \cap W_{\text {loc }}^{1,2}\left(\mathbb{R}^{+} ; L^{2}(\mathbb{T} ; \mathbb{R})\right), \\
w_{0}^{\epsilon} \rightarrow u_{0 x} & \text { in } L^{\infty}(\mathbb{T} ; \mathbb{R}) \text { as } \epsilon \downarrow 0 .
\end{aligned}
$$

In the case where $\left|k^{\prime}(0+)\right|<\infty$ we proceed in exactly the same manner as in [27], the only exception being that one must apply the results in [13] to obtain [27, Proposition 4.1] under the assumptions used here. Hence, we shall concentrate on the case where $\left|k^{\prime}(0+)\right|=\infty$.

By Lemma 3 we know that the functions $w^{\epsilon}$ and $v^{\epsilon}$ are uniformly bounded (at least on compact $t$-intervals) and therefore there exist functions $w$ and $v$ that belong to $L_{\text {loc }}^{\infty}\left(\mathbb{R}^{+} \times \mathbb{T} ; \mathbb{R}\right)$ such that $w^{\epsilon_{j}} \rightarrow w$ and $v^{\epsilon_{j}} \rightarrow v$ in the weak-* sense for some sequence $\epsilon_{j} \downarrow 0$. Furthermore, there exists a function $W$ so that $\sigma\left(w^{\epsilon_{j}}\right) \rightarrow W$ in the weak-* sense as well, and we must show that $W=\sigma(w)$. We shall do this by proving that we actually have strong convergence; that is, we are going to prove that for each $T>0$ we have 


$$
\begin{aligned}
w^{\epsilon_{j}} \rightarrow w, \quad \sigma\left(w^{\epsilon_{j}}\right) \rightarrow \sigma(w), \text { and } v_{j}^{\epsilon} \rightarrow v \\
\\
\operatorname{in~} L^{2}\left([0, T] ; L^{2}(\mathbb{T} ; \mathbb{R})\right) \text { as } j \rightarrow \infty
\end{aligned}
$$

Let $T>0$ be fixed for the moment. We want to apply Lemma 5 and use (14) and therefore we must check that the measure $\beta$ satisfies the necessary conditions.

Since $b$ is nonincreasing and $b(0+)=\infty$ (because we assume that $\left|k^{\prime}(0+)\right|=$ $\infty)$ we automatically get that $\beta([0,1])=\infty$ so it remains to check the requirement on the Fourier transform. (If we knew that $b$ is locally absolutely continuous, e.g., convex, then this requirement would be automatically satisfied.) If $k(0+)<\infty$ one can easily deduce directly from (3) that the function $\bullet a(\bullet)$ is locally absolutely continuous and then it follows from (5) that the function $\bullet b(\bullet)$ is locally absolutely continuous as well and this gives the desired conclusion by the Riemann-Lebesgue lemma. It remains to consider the case where $k(0+)=\infty$ and we show that

$$
\lim _{|\omega| \rightarrow \infty} \omega \hat{b}^{\prime}(i \omega)=0
$$

and this is sufficient as is easy to see. By (19) we have

$$
i \omega \hat{b}^{\prime}(i \omega)=-\frac{1}{\pi} \int_{0}^{\infty} \frac{i \omega \hat{k}(i \omega)+i \omega^{2} \hat{k}^{\prime}(i \omega)}{(\lambda+i \omega \hat{k}(i \omega))^{2} \sqrt{\lambda}} d \lambda
$$

and since the fact that $k$ is nonincreasing implies that $\Re(i \omega \hat{k}(i \omega)) \geq 0$ so that $|\lambda+i \omega \hat{k}(i \omega)| \geq|i \omega \hat{k}(i \omega)|, \omega \in \mathbb{R}$, and $\sup _{\omega \neq 0}\left|\omega \hat{k}^{\prime}(i \omega)\right| /|\hat{k}(i \omega)|<5 \sqrt{2}$ by $[14$, Lemma 6.2.2], we get the desired conclusion because $\lim _{|\omega| \rightarrow \infty}|\omega \hat{k}(i \omega)|=\infty$.

Thus we can apply Lemma 5 and since we assumed that $\sigma^{\prime}$ is bounded, we conclude that there exists a continuous function $\eta$ with $\eta(0)=0$ such that for all $\tau \in[0, T]$

$$
\int_{0}^{T}\left\|\sigma\left(w^{\epsilon}(t+\tau, \bullet)\right)-\sigma\left(w^{\epsilon}(t, \bullet)\right)\right\|_{L^{2}(\mathbb{T})}^{2} d t \leq \eta(\tau)
$$

Define the functions $W^{h}$ and $W^{h, j}$ by

$$
\begin{aligned}
W^{h}(t, x) & =\frac{1}{h} \int_{t}^{t+h} W(s, x) d s, \\
W^{h, j}(t, x) & =\frac{1}{h} \int_{t}^{t+h} \sigma\left(w^{\epsilon_{j}}(s, x)\right) d s .
\end{aligned}
$$

It is clear that $W^{h, j} \rightarrow W^{h}$ in the weak- $*$ sense as $j \rightarrow \infty$, but we claim that we actually have convergence in $L^{2}\left([0, T] ; L^{2}(\mathbb{T} ; \mathbb{R})\right)$. To see that this is the case we note that if we integrate both sides of the second equation in (8) with 
respect to $t$ and $x$ over the rectangle $[t, t+h] \times[x, x+\tau]$, then we get (43)

$$
\begin{aligned}
& \frac{1}{h} \int_{x}^{x+\tau}( \gamma\left(v^{\epsilon_{j}}(t+h, \xi)-g^{\epsilon_{j}}(t+h, \xi)\right) \\
& \quad+\int_{0}^{t+h} b(t+h-s)\left(v^{\epsilon_{j}}(s, \xi)-g^{\epsilon_{j}}(s, \xi)\right) d s \\
&\left.\quad-\gamma\left(v^{\epsilon_{j}}(t, \xi)-g^{\epsilon_{j}}(t, \xi)\right)-\int_{0}^{t} b(t-s)\left(v^{\epsilon_{j}}(s, \xi)-g^{\epsilon_{j}}(s, \xi)\right) d s\right) d \xi \\
&=W^{h, j}(t, x+\tau)-W^{h, j}(t, x) \\
& \quad+\epsilon_{j} \frac{1}{h} \int_{t}^{t+h}\left(v_{x}^{\epsilon_{j}}(s, x+\tau)-v_{x}^{\epsilon_{j}}(s, x)\right) d s .
\end{aligned}
$$

It follows from Hölder's inequality and (13) that

$$
\begin{array}{r}
\int_{-1}^{1}\left|\epsilon_{j} \frac{1}{h} \int_{t}^{t+h}\left(v_{x}^{\epsilon_{j}}(s, x+\tau)-v_{x}^{\epsilon_{j}}(s, x)\right) d s\right|^{2} d x \\
\quad \leq 4 \frac{\epsilon_{j}}{h} \epsilon_{j} \int_{0}^{T+h} \int_{-1}^{1}\left|v_{x}^{\epsilon}(t, x)\right|^{2} d x d t \leq C \frac{\epsilon_{j}}{h},
\end{array}
$$

where $C$ is some constant that does not depend on $\epsilon_{j}$ or $\tau$. Thus if follows from (43) and from the fact that $v^{\epsilon}$ and $g^{\epsilon}$ are bounded that

$$
\lim _{\tau \downarrow 0} \limsup _{j \rightarrow \infty} \int_{-1}^{1}\left|W^{h, j}(t, x+\tau)-W^{h, j}(t, x)\right|^{2} d x=0, \quad t \in[0, T] .
$$

Since the functions $W^{h, j}$ are clearly equicontinuous as functions of $t$ as well, we now get the desired claim that

$$
\lim _{j \rightarrow \infty}\left\|W^{h, j}-W^{h}\right\|_{L^{2}\left([0, T] ; L^{2}(\mathbb{T})\right)}=0,
$$

from the Arzelà-Ascoli theorem.

It is clear that we can choose $\eta$ in such a way that we also have

$$
\int_{0}^{T}\|W(t+\tau, \bullet)-W(t, \bullet)\|_{L^{2}(\mathbb{T})}^{2} d t \leq \eta(\tau) .
$$

Now it follows from (42) and (45) (because we may assume that $\eta$ is nondecreasing) that

$$
\begin{aligned}
\left\|W^{h, j}-\sigma\left(w^{\epsilon_{j}}\right)\right\|_{L^{2}\left([0, T] ; L^{2}(\mathbb{T})\right)} & \leq \sqrt{\eta(h)}, \quad j \geq 1, \\
\left\|W^{h}-W\right\|_{L^{2}\left([0, T] ; L^{2}(\mathbb{T})\right)} & \leq \sqrt{\eta(h)},
\end{aligned}
$$

hence we have by (44) that

$$
\begin{aligned}
& \limsup _{j \rightarrow \infty}\left\|\sigma\left(w^{\epsilon_{j}}\right)-W\right\|_{L^{2}\left([0, T] ; L^{2}(\mathbb{T})\right)} \\
& \quad \leq \quad \underset{j \rightarrow \infty}{\limsup }\left\|\sigma\left(w^{\epsilon_{j}}\right)-W^{h, j}\right\|_{L^{2}\left([0, T] ; L^{2}(\mathbb{T})\right)} \\
& \quad+\limsup _{j \rightarrow \infty}\left\|W^{h, j}-W^{h}\right\|_{L^{2}\left([0, T] ; L^{2}(\mathbb{T})\right)}+\limsup _{j \rightarrow \infty}\left\|W^{h}-W\right\|_{L^{2}\left([0, T] ; L^{2}(\mathbb{T})\right)} \\
& \quad \leq 2 \sqrt{\eta(h)}
\end{aligned}
$$


and we get the desired result that $\sigma\left(w^{\epsilon_{j}}\right) \rightarrow W$ in $L^{2}\left([0, T] ; L^{2}(\mathbb{T} ; \mathbb{R})\right)$. Because $\inf _{s \in \mathbb{R}} \sigma^{\prime}(s)>0$ we conclude that the sequence $w^{\epsilon_{j}}$ must converge strongly as well and it follows that $W=\sigma(w)$. Thus we have obtained the first and second claims of (41). The third part is obtained by almost exactly the same argument as the one used for $\sigma\left(w^{\epsilon_{j}}\right)$, the only difference is that in this case we integrate both sides of the first equation in (8).

It follows directly from Lemma 3 and (40) that the third inequality in (17) holds and that for almost every $t$

$$
\underset{x \in[0,1]}{\operatorname{ess} \sup }\left|\int_{w_{\diamond}}^{w(t, x)} \sqrt{\sigma^{\prime}(s)} d s\right| \leq 2 B(t) .
$$

Let us define the function $u$ by (16) and $u^{\epsilon_{j}}$ by

$$
u^{\epsilon_{j}}(t, x)=\int_{0}^{x} w_{0}^{\epsilon_{j}}(\xi) d \xi+\int_{0}^{t} c(t-s) v^{\epsilon_{j}}(s, x) d s .
$$

If we take the convolution of both sides of the first eqution in (8) with $c$, then we conclude that

$$
w^{\epsilon_{j}}(t, x)=w_{0}^{\epsilon_{j}}(x)+\int_{0}^{t} c(t-s) v_{x}^{\epsilon_{j}}(s, x) d s+\epsilon \int_{0}^{t} c(t-s) w_{x x}^{\epsilon_{j}}(s, x) d s .
$$

If we integrate both sides of this equation over $[0, x]$, use the fact that $v^{\epsilon_{j}}(t, 0)$ $=w_{x}^{\epsilon_{j}}(t, 0)=0$ (because $v^{\epsilon_{j}}$ and $w_{x}^{\epsilon_{j}}$ are odd functions of the second variable), then we conclude with the aid of (13), (40), and (41) that

$$
\int_{0}^{x} w(t, \xi) d \xi=u_{0}(x)+\int_{0}^{t} c(t-s) v(s, x) d s=u(t, x) .
$$

From this equation together with (46) we see that $u \in L_{\text {loc }}^{\infty}\left(\mathbb{R}^{+} ; W_{0}^{1, \infty}([0,1] ; \mathbb{R})\right)$ and that $w=u_{x}$, so that (46) is in fact the first inequality in (17).

If we twice take the convolution of the both sides of the second equation in (8) with $c$ and use (10), then we get

$$
\begin{aligned}
& \int_{0}^{t} c(t-s) v^{\epsilon_{j}}(s, x) d x=\int_{0}^{t} c(t-s) g^{\epsilon_{j}}(s, x) d x \\
& \quad+\int_{0}^{t} \int_{0}^{\tau} k(\tau-s)\left(\left(\sigma\left(w^{\epsilon_{j}}\right)\right)_{x}(s, x)+\epsilon v_{x x}^{\epsilon_{j}}(s, x)\right) d s d \tau,
\end{aligned}
$$

and it follows from (40), (41), the fact that $w=u_{x}$, and from our definitions of $u, g$, and $g^{\epsilon}$ that (15) holds. The remaining claims of Theorem 4 follow easily and the proof is completed.

\section{REFERENCES}

1. D. Brandon and W. J. Hrusa, Global existence of smooth shearing motions of a nonlinear viscoelastic fluid, J. Integral Equations Appl. 2 (1990), 333-351.

2. C. M. Dafermos, Development of singularities in the motion of materials with fading memory, Arch. Rational Mech. Anal. 91 (1986), 193-205.

3. C. M. Dafermos and J. A. Nohel, Energy methods for nonlinear hyperbolic Volterra integrodifferential equations, Comm. Partial Differential Equations 4 (1979), 219-278.

4. W. Desch and R. Grimmer, Smoothing properties of linear Volterra integrodifferential equations, SIAM J. Math. Anal. 20 (1989), 116-132. 
5. H. Engler, Weak solutions of a class of quasilinear hyperbolic integro-differential equations describing viscoelastic materials, Arch. Rational Mech. Anal. 113 (1991), 1-38.

6. Y. Fujita, Integrodifferential equation which interpolates the heat equation and the wave equation, Osaka J. Math. 27 (1990), 309-321.

7. Y. Fujita, Integrodifferential equation which interpolates the heat equation and the wave equation. II, Osaka J. Math. 27 (1990), 797-804.

8. G. Gripenberg, On Volterra equations of the first kind, Integral Equations Operator Theory 3/4 (1980), 473-488.

9. - Nonexistence of smooth solutions for shearing flows in a nonlinear viscoelastic fluid, SIAM J. Math. Anal. 13 (1982), 954-961.

10. — Volterra integro-differential equations with accretive nonlinearity, J. Differential Equations 60 (1985), 57-79.

11. - Global existence of solutions of Volterra integrodifferential equations of parabolic type, J. Differential Equations 102 (1993), 382-390.

12. - Nonlinear Volterra equations of parabolic type due to singular kernels, J. Differential Equations (to appear).

13. - Compensated compactness and one-dimensional elastodynamics, manuscript.

14. G. Gripenberg, S-O. Londen, and O. Staffans, Volterra integral and functional equations, Cambridge University Press, Cambridge, 1990.

15. H. Hattori, Breakdown of smooth solutions in dissipative nonlinear hyperbolic equations, Quart. Appl. Math. 40 (1982), 113-127.

16. W. J. Hrusa, Global existence and asymptotic stability for semilinear hyperbolic Volterra equation with large initial data, SIAM J. Math. Anal. 16 (1985), 110-134.

17. W. J. Hrusa and J. A. Nohel, The Cauchy problem in one-dimensional nonlinear viscoelasticity, J. Differential Equations 59 (1985), 388-412.

18. W. J. Hrusa, J. A. Nohel, and M. Renardy, Initial value problems in viscoelasticity, Appl. Mech. Rev. 41 (1988), 371-378.

19. W. J. Hrusa and M. Renardy, On wave propagation in linear viscoelasticity, Quart. Appl. Math. 43 (1985), 237-254.

20. - On a class of quasilinear partial integrodifferential equations with singular kernels, J. Differential Equations 64 (1986), 195-220.

21. - A model equation for viscoelasticity with a strongly singular kernel, SIAM J. Math. Anal. 19 (1988), 257-269.

22. S-O. Londen, An existence result on a Volterra equation in a Banach space, Trans. Amer. Math. Soc. 235 (1978), 285-304.

23. - On an integrodifferential Volterra equation with a maximal monotone mapping, J. Differential Equations 27 (1978), 405-420.

24. - Some existence results for a nonlinear hyperbolic integrodifferential equation with singular kernel, J. Integral Equations Appl. 3 (1991), 3-30.

25. R. C. MacCamy, A model for one-dimensional nonlinear viscoelasticity, Quart. Appl. Math. 35 (1977), 21-33.

26. J. A. Nohel and M. Renardy, Development of singularities in nonlinear viscoelasticity, Amorphous Polymers and Non-Newtonian Fluids (C. Dafermos, J. L. Ericksen, and D. Kinderlehrer, eds.), IMA Math. Appl., Vol. 6, Springer-Verlag, Berlin and New York, 1987, pp. 139-152.

27. J. A. Nohel, R. C. Rogers, and A. E. Tzavaras, Weak solutions for a nonlinear system in viscoelasticity, Comm. Partial Differential Equations 13 (1988), 97-127.

28. J. Prüss, Positivity and regularity of hyperbolic Volterra equations in Banach spaces, Math. Ann. 279 (1987), 317-344.

29. J. Prüss, Quasilinear parabolic Volterra equations in spaces of integrable functions, Semigroup Theory and Evolution Equations: The Second International Conference $(\mathrm{Ph}$. Clement, E. Mitidieri, and B. de Pagter, eds.), Lecture Notes in Pure and Appl. Math., vol. 135, Dekker, New York, 1991, pp. 401-420. 
30. M. Renardy, Coercive estimates and existence of solutions for a model of one-dimensional viscoelasticity with a non-integrable memory function, J. Integral Equations Appl. 1 (1988), 7-16.

31. M. Renardy, W. J. Hrusa, and J. A. Nohel, Mathematical problems in viscoelasticity, Longman, London, 1987.

32. O. Staffans, On a nonlinear hyperbolic Volterra equation, SIAM J. Math. Anal. 11 (1980), 793-812.

Department of Mathematics, P. O. Box 4, 00014 University of Helsinki, Finland

E-mail address: Gustaf . Gripenberg@helsinki.fi 